\title{
SIMPLIFIED MODELS OF ENGINE HC EMISSIONS, EXHAUST TEMPERATURE AND CATALYST TEMPERATURE FOR AUTOMOTIVE COLDSTART
}

\author{
J.Carlos Zavala ${ }^{*, 1}$ Pannag R. Sanketi* \\ M.Wilcutts* T.Kaga** J. K. Hedrick* \\ * Vehicle Dynamics Laboratory, Etcheverry Hall, \\ University of California, Berkeley, USA-94720 \\ ** Toyota Technical Center U.S.A., Inc. Berkeley \\ Laboratory, Tech. Research Dept.
}

\begin{abstract}
The initial 1-2 minutes of operation of an automotive spark-ignition engine, commonly called as the "coldstart" period, produces more than 75-80 $\%$ of the hydrocarbon $(\mathrm{HC})$ emissions in a typical drive cycle. Model-based controller development requires accurate, yet simple, models that can run in realtime. Simple, intuitive models are developed to predict both tailpipe hydrocarbon (HC) emissions and exhaust temperature during coldstart. Each of the models is chosen to be sum of first order linear systems based on the experimental data and ease of parameter identification. Inputs to these models are $A F R$, spark timing and engine crankshaft speed. A reduced order thermodynamic model for the catalyst temperature is also developed. The parameters are identified using least squares technique. The model estimates for the coldstart are compared with the experimental results with good agreement. Submitted to Fifth IFAC Symposium on Advances in Automotive Control
\end{abstract}

Keywords: Coldstart, Engine Thermal Model, Catalyst Model

\section{INTRODUCTION}

As regulations on emissions for automotive engines become more stringent, the reduction of hydrocarbon ( $\mathrm{HC}$ ) emissions during coldstart continues to pose a relevant challenge for the industry. Developing simple models which can run in realtime is necessary to design controllers using the model-based paradigm.

A lot of interest has been shown in modeling and analysis of engine and catalyst subsystems for coldstart analysis. The initial work on physically based dynamic engine models that account for the

\footnotetext{
1 czavala@me.berkeley.edu
}

throttle dynamics and intake manifold dynamics can be traced back to (Powell 1979) and (Dobner 1983). It was further modified by (Moskwa and Hedrick 1987) and (Cho and Hedrick 1989). Recent work on engine subsytems modeling can be found in (Tunestal and Hedrick 2001), (Balluchi et al. 2001),(Shaw and Hedrick 2002), (Zavala et al. 2006),(Sanketi et al. 2006b). (Shaw and Hedrick 2002) and references therein developed models for engine-out exhaust gas temperature $\left(T_{e x h}\right)$ and raw $\mathrm{HC}\left(H C_{\text {raw }}\right)$. In this paper, simple, intuitive models for $T_{e x h}$ and $H C_{\text {raw }}$ are developed. Each of these is modeled as a sum of first order linear systems based on the experimental data and ease of parameter identification. Inputs to these models are air to fuel ratio $(A F R)$, spark 
timing and engine crankshaft speed $\left(\omega_{e}\right)$. AFR, spark and changes in $\omega_{e}$ are related to the combustion stability. (See Sec. 2.1 and 2.2).

Modeling and analysis of catalysts is also important for coldstart controller design. These range from very complex ones to simplified ones which are more suitable for closed-loop control. Some of the important contributions are (Ohsawa et al. 1998), (Shen et al. 1999), (Chan and Hoang 1999), (Jones et al. 2000), (Brandt et al. 2000),(Fiengo et al. 2002), (Shaw et al. 2002), (Koltsakis and Tsinoglou 2003), (Sanketi et al. 2006a) and references therein. The catalyst model presented here is an improved version of the model developed in (Sanketi et al. 2006a). A thermodynamic energy balance is used to model the catalyst temperature $\left(T_{\text {cat }}\right)$. Further, thermal diffusivity calculation is used to reduce the order of the catalyst temperature model. (See Sec. 2.3).

The contribution of this paper lies in developing new simplified models for $H C_{\text {raw }}, T_{\text {exh }}$ and $T_{\text {cat }}$. Our goal is to develop a simplified model-based controller for coldstart. A MIMO sliding controller for coldstart designed using these models is presented in another paper submitted to the same symposium.

\section{MODEL OF PLANT}

The model presented here has been developed based on the influence of the $A F R$, engine speed and spark timing on $T_{\text {exh }}$ and $H C_{\text {raw }}$. $A F R$ and spark timing directly affect the combustion process. In addition, changes in engine speed affect the cylinder pressure and hence, the quality of combustion. Experiments indicated that the engine speed was having a more noticeable influence on $H C_{\text {raw }}$ and $T_{e x h}$ as compared to the intake air flow rate. Also, each of the chosen inputs can be modified easily to observe the effect on $T_{e x h}$, which simplifies the process of modeling. The $T_{\text {exh }}, H C_{\text {raw }}$ and $T_{\text {cat }}$ submodels have been simulated together with an engine model presented in (Cho and Hedrick 1989) and (Sanketi et al. 2006b).

\subsection{Exhaust Temperature Model}

Through experiments, it was observed that $T_{e x h}$ was responding almost like a first order linear system to each of the input variables. Hence, the output was modeled as a set of three first order linear systems running in parallel that relate the three variables mentioned before to $T_{\text {exh }}$. The block diagram shown in Fig.1 illustrates the idea. It should be noted that however, the inputs have been offset around certain nominal value

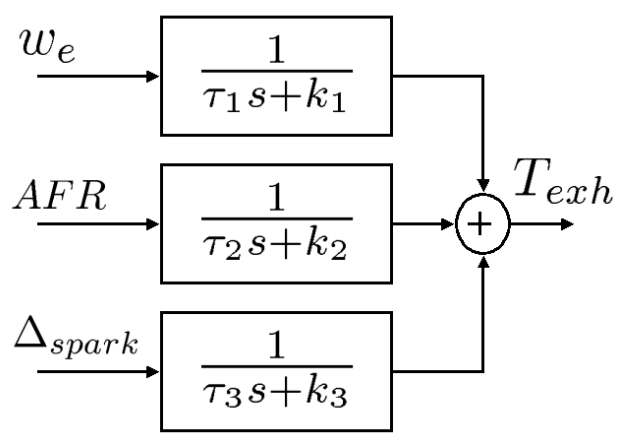

Fig. 1. Block Model for Exhaust temperature model

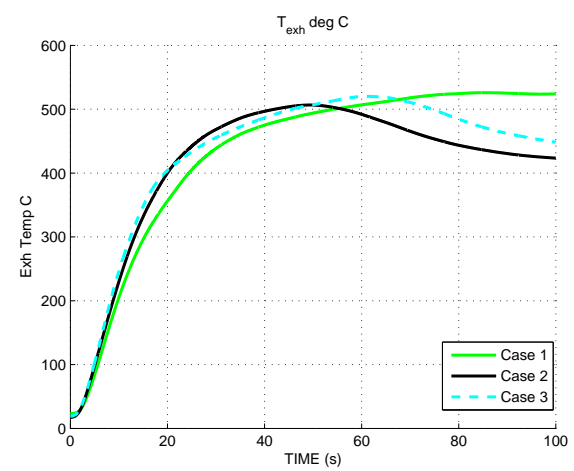

Fig. 2. Experiments showing the effect of inputs on exhaust temperature- $T_{\text {exh }}$

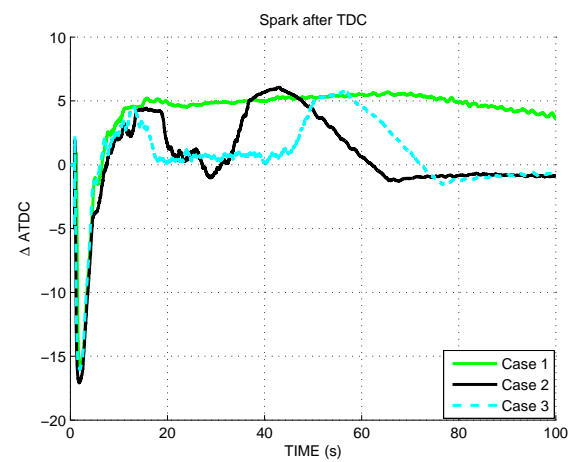

Fig. 3. Experiments showing the effect of inputs on exhaust temperature- Spark

in the model and saturations have been applied, too. Negative actuation has also to be ruled out. Though this model does not follow any physicsbased combustion modeling, it is intuitive; and it is simple and suitable for engine controller design.

Figures 2 through 5 illustrate the effect of inputs on $T_{\text {exh }}$. Retarding the spark, increasing the engine speed and a richer $A F R$ lead to an increase in $T_{e x h} . T_{e x h}$ is slower in response to $A F R$ and engine speed as compared to the spark timing. Also, the effect of spark timing was found to be more prominent than that of others. The parameters of the model were obtained for the coldstart period using least squares. 


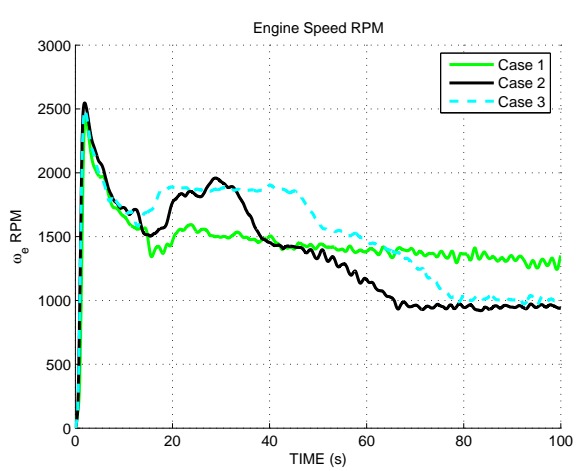

Fig. 4. Experiments showing the effect of inputs on exhaust temperature- RPM

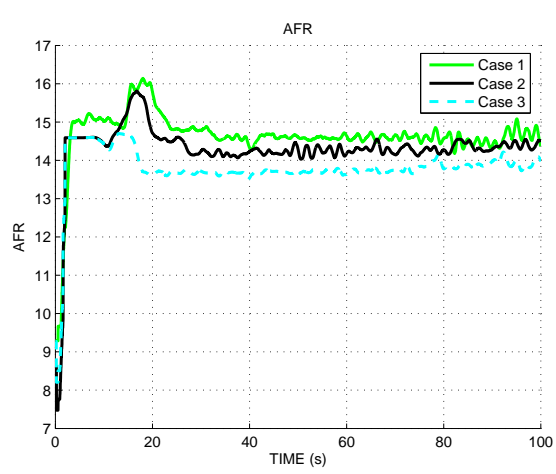

Fig. 5. Experiments showing the effect of inputs on exhaust temperature- AFR
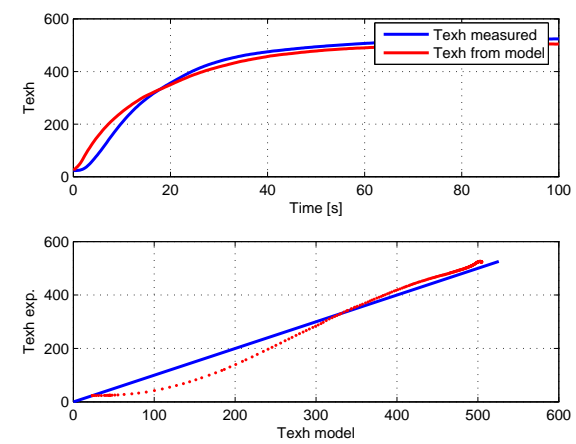

Fig. 6. Comparison between measured and computed exhaust temperature for a coldstart run

Fig.6 shows the comparison of the model versus experimental data for an engine coldstart run. The second part of the figure shows an error of about $50 \mathrm{C}$, however, the first part of the figure illustrates that it is due to a small delay in the transient rise of the temperature. This will not have a significant effect on the catalyst efficiency profile. Fig. 7 shows the comparison between the model and the experimental values for exhaust temperature over several coldstart experiments. Errors of about 50-60 $C$ are consistently found in the transient part of the temperature profile. A steady state error of about $80 \mathrm{C}$ is also found in one of the data sets. Overall, the mean error was

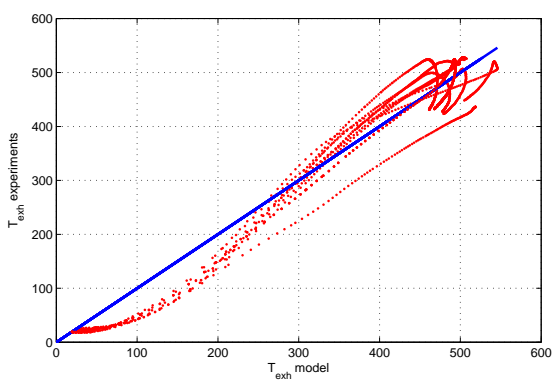

Fig. 7. Comparison between measured and computed exhaust temperature for several coldstart runs

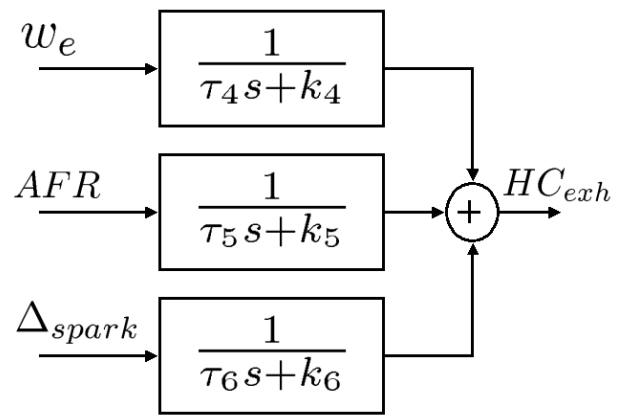

Fig. 8. Block Model for hydrocarbon emissions model

$4.9414 C$ with a standard deviation of $34.8981 C$.

\subsection{Hydrocarbon Emissions Model}

The raw hydrocarbon emissions $H C_{\text {raw }}$ was also modeled as a sum of first-order linear systems, very similar in concept to that of $T_{e x h}$. It makes sense since both $H C_{\text {raw }}$ and $T_{e x h}$ are products of the same process. As in the case of $T_{e x h}$, three linear subsystems describe the interaction between $A F R$, engine speed and spark timing with the hydrocarbon emissions. However, the input offsets are different than those used for the $T_{\text {exh }}$ model. Fig.8 illustrates the concept of the model.

Figures 9 through 12 illustrate the effect of inputs on $H C_{\text {raw }}$. Retarding the spark above certain value, decreasing the engine speed and a richer $A F R$ lead to an increase in $H C_{\text {raw }}$. $H C_{\text {raw }}$ is much faster in response to $A F R$ as compared to the engine speed and the spark timing. Also, the effect of $A F R$ was found to be more prominent than others. The parameters of the model were obtained for the coldstart period using least squares.

Fig.13 shows the comparison between the model and the experimental hydrocarbon emissions for a coldstart run and Fig.14 shows the comparison of the model and experimental hydrocarbon 


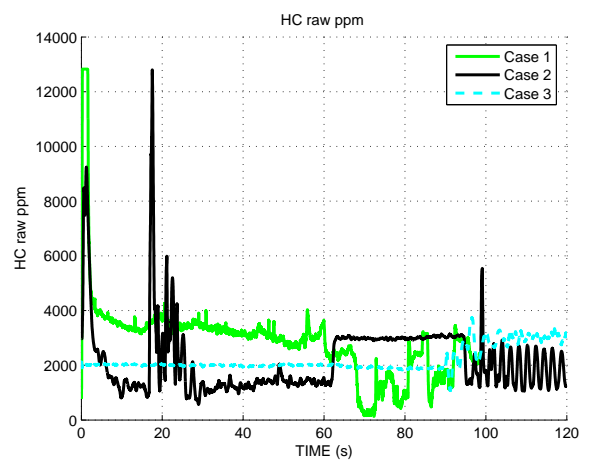

Fig. 9. Experiments showing the effect of inputs on raw emissions- $H C_{\text {raw }}$

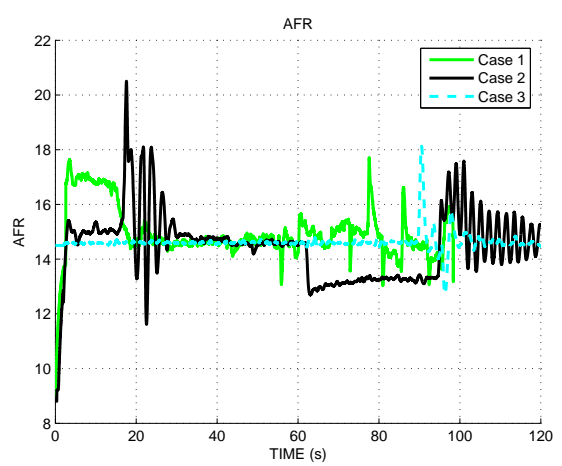

Fig. 10. Experiments showing the effect of inputs on raw emissions- $A F R$

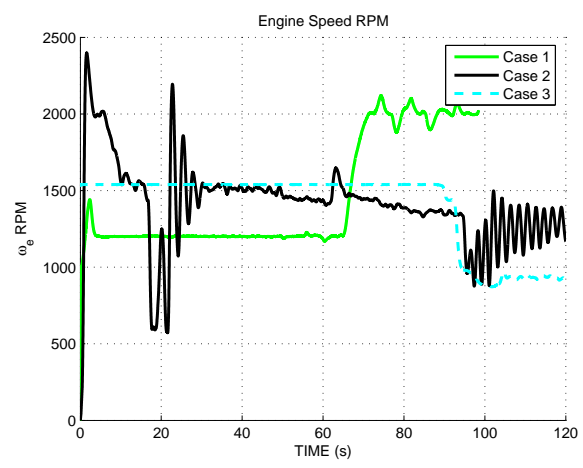

Fig. 11. Experiments showing the effect of inputs on raw emissions- $R P M$

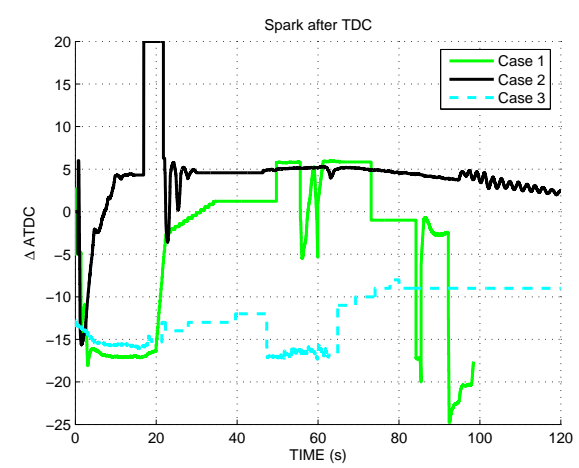

Fig. 12. Experiments showing the effect of inputs on raw emissions- Spark
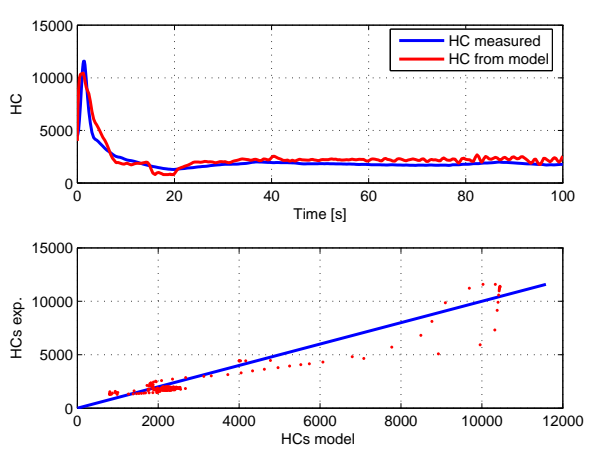

Fig. 13. Comparison between measured and computed hydrocarbon for one coldstart run

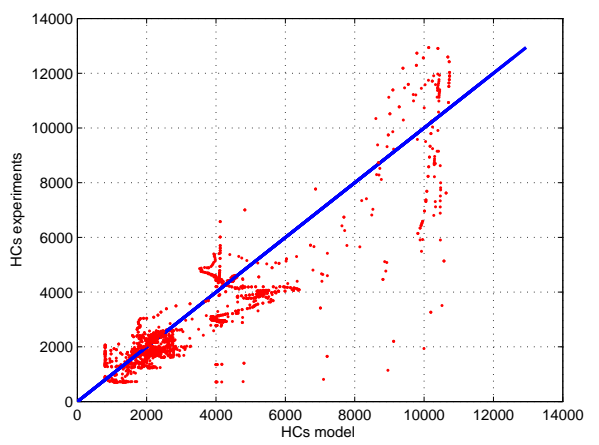

Fig. 14. Comparison between measured and computed hydrocarbon for several coldstart runs

emissions in ppm over several experiments. The largest errors occur during the transients close to the peak of the emissions which is the period when the signal changes more rapidly, whereas the errors are lower at steady state. Overall, the mean error was $388.7233 \mathrm{ppm}$ with a standard deviation of $959.3685 \mathrm{ppm}$.

\subsection{Catalyst Model}

This part describes the physics that governs the catalyst warm up including the water condensation and evaporation effect. The simplified catalyst model has a structure similar to that described in (Sanketi et al. 2006a). Here, a thermal diffusivity calculation is used to show that the gas comes in equilibrium with the solid before it leaves the catalyst, thus making unnecessary the use of different states for the catalyst solid temperature and the gas temperature.

A single cell of the catalytic converter is analyzed. The time scale required for the gas and the honeycomb mass to come in equilibrium is given by

$$
t_{\text {diff }}=\frac{d^{2}}{\alpha}
$$

where, the characteristic length $d$ for diffusion is equal to half the cell side length and $\alpha$ is thermal diffusivity of the gas. This was compared with 
the time required for the gas to travel through the catalyst, which was calculated using the average exhaust gas flow rate in a typical coldstart. The calculations were performed at three different temperatures spanning the coldstart. The results are shown in Table 1.

Table 1. Time for travel and diffusion time scale

\begin{tabular}{llll}
\hline No. & $\begin{array}{l}\text { Gas Temp. } \\
(\mathrm{K})\end{array}$ & $\begin{array}{l}\text { Time Travel } \\
(\mathrm{s})\end{array}$ & $\begin{array}{l}\text { Diffusion Time } \\
\text { Scale }(\mathrm{s})\end{array}$ \\
\hline 1 & 350 & 0.1114 & 0.0559 \\
2 & 550 & 0.0709 & 0.0269 \\
3 & 700 & 0.0557 & 0.0183 \\
\hline
\end{tabular}

From table 1, it can be seen that the time taken by the gas to travel through the catalyst is two to three times the time scale for the gas to come into equilibrium with the catalyst solid mass. Hence, for our analysis, we can assume that the gas and the solid mass of the catalyst are at the same temperature and modeling the catalyst requires only one temperature state instead of two.

\subsection{Model Equations}

The catalyst temperature depends on the heat obtained from the feedgas flowing into the catalyst, the amount of heat generated due to oxidation of pollutants from the feedgas $\left(\dot{Q}_{g e n}\right)$ and the heat transfer to the surroundings $\left(\dot{Q}_{\text {out }}\right)$. In addition to these heat transfers, heat is lost due to evaporation of the moisture in the catalyst $\left(\dot{Q}_{e v}\right)$. This moisture is accumulated in the initial few seconds of operation due to condensation of vapor on the cold surface of the catalytic converter. When the temperature reaches the saturation temperature of the condensed vapor, the vapor starts evaporating. Due to the heavy heat loss in evaporation, the catalyst temperature remains almost constant or increases at a very low rate. Thus, there is a plateau in the temperature profile. The catalyst temperature dynamics can be summarized as follows.

During the plateau:

$$
\begin{array}{r}
\dot{T}_{c a t}=\frac{1}{m_{g a s} C_{v}+M C}\left[\dot{m}_{e x h} C_{p}\left(T_{e x h}-T_{t p}\right)\right. \\
\left.-\dot{Q}_{o u t}+\dot{Q}_{g e n}-\dot{Q}_{e v}\right]
\end{array}
$$

otherwise

$$
\begin{gathered}
\dot{T}_{c a t}=\frac{1}{m_{g a s} C_{v}+M C}\left[\dot{m}_{e x h} C_{p}\left(T_{e x h}-T_{t p}\right)\right. \\
\left.-\dot{Q}_{o u t}+\dot{Q}_{g e n}\right] \\
\dot{Q}_{e v}=\dot{m}_{e v} \Delta \bar{h}_{e v}
\end{gathered}
$$
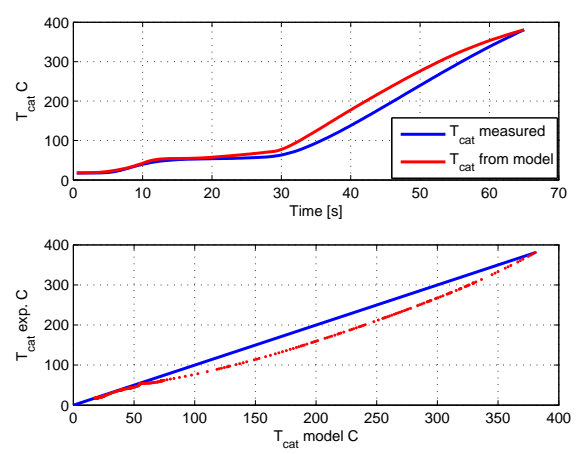

Fig. 15. Comparison between measured and computed $T_{\text {cat }}$ for several coldstart runs

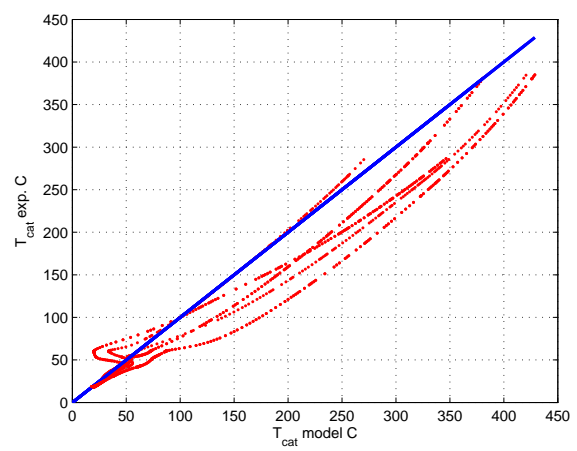

Fig. 16. Comparison between measured and computed $T_{\text {cat }}$ for several coldstart runs

where, $\dot{m}_{e x h}$ is the exhaust gas flow rate, $M$ is the solid mass of the catalyst, $m_{\text {gas }}$ is the mass of the gas inside the catalyst, $\dot{m}_{e v}$ is the mass rate of evaporation and $\Delta \bar{h}_{e v}$ is the enthalpy of evaporation at the corresponding saturation temperature. It is not straightforward to find $\dot{Q}_{e v}$ directly. Hence, it is calculated implicitly using a method that is similar to that used in (Sanketi et al. 2006a). It employs a parameter adaptation algorithm that uses the $T_{\text {cat }}$ measurement to find $\dot{Q}_{\text {ev }}$ through $\dot{Q}_{\text {out }}$. Readers are encouraged to read (Sanketi et al. 2006a) for details.

Fig.15 shows the comparison of the model versus experimental data for an engine coldstart run. Fig.16 shows the comparison between the model and the experimental values for exhaust temperature over several coldstart experiments. Overall, the mean error was $10.4227 C$ with a standard deviation of $22.2439 C$.

\section{CONCLUSIONS}

Simplified control-oriented models of engine-out hydrocarbon emissions $\left(H C_{\text {raw }}\right)$, exhaust temperature $\left(T_{e x h}\right)$, and catalyst temperature $\left(T_{c a t}\right)$ were presented. $H C_{\text {raw }}$ was modeled as a sum of first order linear systems with three different inputs namely, air-fuel ratio $A F R$, spark timing and engine speed $\omega_{e}$. Such a model structure was based 
on the experimental data and ease of parameter identification. The structure of the $T_{e x h}$ model was chosen similar to that of $H C_{\text {raw }}$. A reduced order thermodynamic model for the catalyst temperature was developed using thermal diffusivity analysis. The parameters were identified using least squares methodology. The model estimates during the warm-up agreed well with the experimental results. A MIMO sliding controller for coldstart designed using these models is presented in another paper submitted to the same symposium.

\section{ACKNOWLEDGEMENTS}

Authors acknowledge the financial support provided by CONACYT (Consejo Nacional de Ciencia y Tecnología de Mexico) and by the Center for Hybrid and Embedded Software Systems (CHESS) at UC Berkeley, which receives support from the National Science Foundation (NSF award \#CCR-0225610), the State of California Micro Program, and the following companies: Agilent, Bosch, DGIST, General Motors, Hewlett Packard, Infineon, Microsoft, National Instruments, and Toyota.

\section{REFERENCES}

Balluchi, A., M. D. Di Benedetto, C. Pinello and A. L. Sangiovanni-Vincentelli (2001). Mixed models of computation in the design of automotive engine control. In: Proc. 40th IEEE Conference on Decision and Control, (Orlando, Florida). (invited paper).

Brandt, E., Y. Wang and J.W. Grizzle (2000). Dynamic modeling of three-way catalyst for si engine exhaust emission control. IEEE Transactions on Control Systems Technology 8(5), 767-776.

Chan, S.H. and D.L. Hoang (1999). Modeling of catalytic conversion of $\mathrm{co} / \mathrm{ch}$ in gasoline exhaust at engine cold-start. SAE Technical Paper 1999-01-0452.

Cho, D. and J. K. Hedrick (1989). Automotive powertrain modelling for control. Journal of Dynamic Systems, Measurements and Control 111, 568-576.

Dobner, D.J. (1983). Dynamic engine models for. control development-part i: Nonlinear and linear model formulation. International Journal of Vehicle Design SP4, 54-74.

Fiengo, G., L. Glielmo, S. Santini and G. Serra (2002). Control oriented models for twcequipped spark ignition engines during the warm-up phase. Proceedings of the American Control Conference pp. 1761-1766.
Jones, J. Peyton, J.B. Roberts and P. Bernard (2000). A simplified model for the dynamics of a three-way catalytic converter. SAE Technical Paper 2000-01-0652.

Koltsakis, G. C. and D. N. Tsinoglou (2003). Thermal response of close-coupled catalysts during light-off. SAE Technical Paper 200301-1876.

Moskwa, J.J. and J. K. Hedrick (1987). Automotive engine modeling for real-time control application. Proceedings of American Control Conference pp. 341-346.

Ohsawa, K., N. Baba and Shinji Kojima (1998). Numerical prediction of transient conversion characteristics in a three-way catalytic converter. SAE Technical Paper 982556.

Powell, B.K. (1979). A dynamic model for automotive engine control analysis. Proc. of IEEE Conf. on Decision and Control pp. 120-126.

Sanketi, P.R., J. Carlos Zavala, J. K. Hedrick, M. Wilcutts and T. Kaga (2006a). A simplified catalytic converter model for automotive coldstart applications with adaptive parameter fitting. 8th International Symposium on Advanced Vehicle Control.

Sanketi, P.R., J.C. Zavala and J.K. Hedrick $(2006 b)$. Automotive engine hybrid modeling and control for reduction of hydrocarbon emissions. International Journal of Control 79(5), 449-464.

Shaw, B. and J. K. Hedrick (2002). Coldstart engine combustion modelling to control hydrocarbon emissions. Proceedings of 15th Triennial World Congress of the International Federation of Automatic Control.

Shaw, B., Gerald D. Fischer and J. K. Hedrick (2002). A simplified coldstart catalyst thermal model to reduce hydrocarbon emissions. Proceedings of 15th Triennial World Congress of the International Federation of Automatic Control.

Shen, H., T. Shamim and S. Sengupta (1999). An investigation of catalytic converter performances during cold starts. SAE Technical Paper 1999-01-3473.

Tunestal, P. and J. K. Hedrick (2001). Cylinder air/fuel ratio estimation using net heat release data. 3rd IFAC Workshop on Advances in Automotive Control.

Zavala, J.C., D. Günther, P.R. Sanketi, M. Wilcutts and K. Hedrick (2006). Fuel dynamics model for coldstart. Proceedings of ASME IMECE 2006. 